\title{
Molecules and dust in the ejecta of Type II-P supernovae
}

\author{
I. Cherchneff and A. Sarangi \\ Department Physik, Universität Basel, Klingelbergstrasse 82, CH-4056 Basel, Switzerland
}

\begin{abstract}
We study the formation of molecules and dust clusters in the ejecta of solar metallicity, Type II-P supernovae using a chemical kinetic approach and follow the evolution of molecules and small dust cluster masses from day 100 to day 1500 after explosion. We predict that large masses of molecules including $\mathrm{CO}, \mathrm{SiO}, \mathrm{SiS}, \mathrm{O}_{2}$, and $\mathrm{SO}$ form in the ejecta. We show that the non-equilibrium chemistry results in a gradual build up of the dust mass from small $\left(\sim 10^{-5}\right.$ $\left.M_{\odot}\right)$ to large values $\left(\sim 5 \times 10^{-2} M_{\odot}\right)$ over a five-year period after explosion. This result provides a natural explanation to the discrepancy between the small dust masses detected at infrared wavelengths some 500 days post-explosion and the larger amounts of dust recently detected with the Herschel telescope in supernova remnants.
\end{abstract}

Keywords. supernovae: general, molecular processes, dust, circumstellar matter, astrochemistry

\section{Introduction}

Massive stars $\left(8 M_{\odot}<\mathrm{M}_{\star}<30 M_{\odot}\right)$ end their life as Core-Collapse supernovae (hereafter SNe) of Type II-P. Despite the huge amount of energy released by the explosion $\left(\sim 1 \times 10^{51} \mathrm{erg}\right)$, and the harsh physical conditions that characterise the ejected stellar gas, dust and molecules have been detected in many SNe, including SN1987A, some hundred days after explosion (e.g., Spyromilio et al. 1988, Lucy et al. 1989, Kotak et al. 2009, Sugerman et al. 2006). The fundamental band of $\mathrm{SiO}$ has been detected in several SNe, e.g., SN2004et, and the fading of the transition over time was ascribed to the depletion of $\mathrm{SiO}$ in the condensation process of silicates at $\sim 400$ days post-outburst (Kotak et al. 2009). Most important are the small masses of warm dust derived with values that range from $1 \times 10^{-5} M_{\odot}$ to $1 \times 10^{-3} M_{\odot}$. The latest data on $\mathrm{SN}$ remnants obtained with the submilimetre (submm) Herschel telescope have cast a new light on the dust released by SN events. A large mass of cold ejecta dust amounting to $0.08 M_{\odot}$ was derived in the 330-year-old SNR Cas A (Barlow et al. 2010). In the Crab Nebula, cool dust was recently detected in the filaments and the derived dust masses amount to 0.1 - $0.24 M_{\odot}$, depending on the type of dust assumed (Gomez et al. 2012). Finally, 0.4 to $0.7 M_{\odot}$ of cool, ejecta dust have been inferred from submm flux data in the young remnant SN1987A (Matsuura et al. 2011). The dust mass produced by SNe is thus uncertain. Here we present the results of physico-chemical models of dust synthesis in Type II-P SNe.

\section{Ejecta physical and chemical model}

A stellar progenitor of mass $15 M_{\odot}$, typical of Type II-P SNe is considered. The stratified ejecta is described by the mass zones of the progenitor core given by explosion models, and we assume that the gas within each zone is fully-microscopically mixed. No gas leakage between different zones is considered. The initial composition of the ejecta in the form of the elemental mass yields are taken from Rauscher et al. (2002). The basic model parameters are similar to those characterising the $20 M_{\odot}$ progenitor of Cherchneff 
\& Dwek $(2009,2010)$, i.e., an explosion energy of $1 \times 10^{51} \mathrm{ergs}$, an effective $\gamma$-ray optical depth at 100 days $\tau(100)=23.8$, and a ${ }^{56} \mathrm{Ni}$ mass of $0.075 M_{\odot}$. The temperature and density dependance as a function of time are given by Sarangi \& Cherchneff (2013).

All possible atoms, molecules, and ions that form in the SN ejecta are considered in our chemical scheme, and we model the ejecta chemistry considering all possible types of chemical reactions relevant to hot and dense environments. All chemical pathways that lead to the formation of linear molecules, carbon chains and rings, and small dust clusters include neutral-neutral processes such as termolecular, bimolecular, radiative association, and charge exchange reactions, whereas the destruction is described by thermal fragmentation, neutral-neutral processes (i.e., oxidation reactions of carbon chains and all reverse processes of the formation reactions), ion-molecule recombination processes and charge exchange reactions. Furthermore, the radioactive decay of ${ }^{56} \mathrm{Ni}$ to ${ }^{56} \mathrm{Co}$, and ${ }^{56} \mathrm{Co}$ to ${ }^{56} \mathrm{Fe}$ creates $\gamma$-rays that degrade to $\mathrm{X}$ rays and UV photons through collision with thermal electrons and triggers the creation of a population of Compton electrons in the gas. These fast electrons ionise atoms and destroy chemical species in the ejecta. The description of the growth pathways of small silicate clusters, namely forsterite dimer $\left(\mathrm{Mg}_{2} \mathrm{SiO}_{4}\right)_{2}$ and enstatite dimer $\left(\mathrm{MgSiO}_{3}\right)_{2}$, is based on the work by Goumans \& Bromley (2012), where the growth of enstatite and forsterite clusters from the $\mathrm{SiO}$ dimer is described by one oxygen-addition step followed by one Mg-inclusion as a recurrent growth scenario.

\section{Results}

The chemistry is followed from day 100 until day 1500, a time span which covers the initial formation of molecules at early times until the dust cluster synthesis is fully completed some 4 years after outburst. We find that the zones of the He-core are efficient at forming large amounts of molecules. Because the ejecta is assumed to be hydrogen-free, the number of chemical species formed is limited, and this poor chemistry typical of SN ejecta is well exemplified by the detection of only two molecules, $\mathrm{CO}$ and $\mathrm{SiO}$, in several SNe (e.g., Lucy et al. 1989, Kotak et al. 2009), and CO in SN remnants (Rho et al. 2012). The mass of silicon monoxide, $\mathrm{SiO}$, is shown in Fig. 1 as a function of post-explosion time for all the various zones in the ejecta. Masses derived from infrared (IR) observations of a few Type II-P SNe are also shown. The SiO mass follows a rapid increase at day 200 in zones $1 \mathrm{~B}, 2$, and 3, when the formation of $\mathrm{SiO}$ is delayed to 400 days in zones $4 \mathrm{~A}$ and $4 \mathrm{~B}$. The prevalent formation processes are radiative association and reaction of atomic $\mathrm{Si}$ with dioxygen, $\mathrm{O}_{2}$, while destruction is mainly due to reactions with $\mathrm{Ar}^{+}$and $\mathrm{Ne}^{+}$. The sharp decrease after day 200 is due to the conversion of $\mathrm{SiO}$ into $\mathrm{SiO}$ dimers and silica and silicate cluster growth.

The evolution of CO mass with post-explosion time for the He-core zones is shown in Fig. 2. CO masses derived from available observational data for SN1987A are also plotted for early times (Liu \& Dalgarno 1995). In zones 4A, 4B, 2 and 3, CO forms as early as 200 days to reach masses ranging from $10^{-4}$ to $10^{-2} M_{\odot}$ at day 1500 . The total ejected CO mass at day 1500 is as large as $\sim 0.1 M_{\odot}$, that is, much larger than the masses derived from IR data before day 600 in SN1987A. These large amounts of CO primarily form in zones $4 \mathrm{~A}$ and $4 \mathrm{~B}$ but do not trace efficient carbon dust formation in these two zones. These zones indeed form no carbon dust because of the quick destruction of carbon chains by reaction with $\mathrm{O}_{2}$ to form CO.

Three other molecules are present in the ejecta, namely SiS, $\mathrm{O}_{2}$ and SO. The gas-phase chemistries of all molecular species and dust clusters are entangled and the ejecta is made of a molecular component equivalent to $\sim 29 \%$ of the $2.5 M_{\odot}$ of ejected material. 


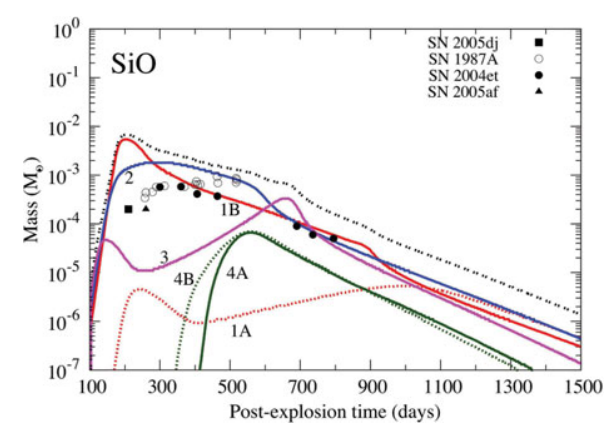

Figure 1. SiO masse variation with post-explosion time for the $15 M_{\odot}$ progenitor as a function of ejecta zones (taken from Sarangi \& Cherchneff 2013). The masses derived from observational data of several $\mathrm{SNe}$ are also shown.

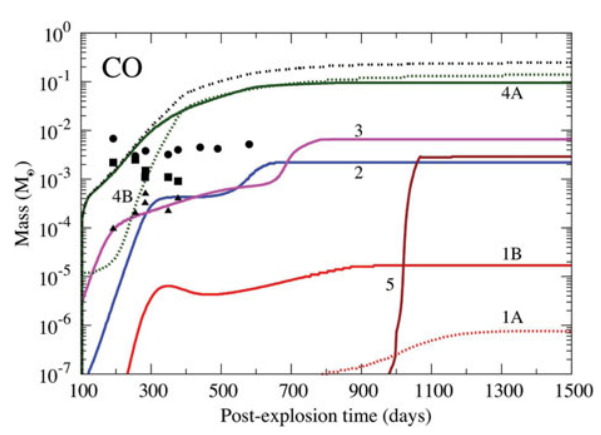

Figure 2. CO mass variation with post-explosion time for the $15 M_{\odot}$ progenitor as a function of ejecta zones (from Sarangi \& Cherchneff 2013). CO masses derived from observations of SN1987A are also shown.

The variation of dust cluster masses summed over all ejecta zones with time is illustrated in Fig. 3. The timing of dust production highly depends on the local chemistry characterising the zones, as exemplified by the formation of silicate and carbon clusters. Forsterite first nucleates at 300 days in zone 1B, and gradually grows to reach its maximum value at $\sim 600$ days in zone 2 . These two nucleation phases are seen as two $\mathrm{SiO}$ depletion events in Figure 1. The gradual growth of the forsterite total mass results from the chemistry of $\mathrm{SiO}$ formation and the growth of silicate clusters. The scenario is different for carbon clusters. Carbon chains grow in significant amounts in the only carbon-rich zone of the ejecta, zone 5. This zone is helium-rich and He atoms are ionised by Compton electrons. The produced ions are destroyed by recombination to $\mathrm{He}$ and the decrease of Compton electrons with time. $\mathrm{He}^{+}$is detrimental to the formation and survival of molecules in zone 5 (Cherchneff \& Dwek 2009), as the ion quickly destroys molecules and postpones the time for carbon chain synthesis.

This sequence of epochs at which dust of various kinds condenses in the ejecta results in a gradual dust mass growth from $\sim 10^{-4} M_{\odot}$ at 400 days to $5 \times 10^{-2} M_{\odot}$ at day 1500. This growth sequence provides a possible explanation to the discrepancy between the mass of warm dust derived at early time from IR data and the mass of cool dust inferred from submm fluxes in SN remnants. Because dust cluster masses represent upper limits to the total dust mass formed in the ejecta, we conclude that $\mathrm{SNe}$ are efficient but moderate dust suppliers to galaxies.

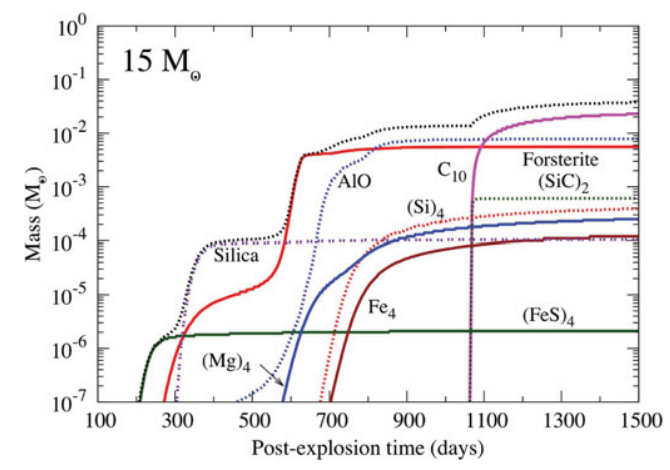

Figure 3. Dust cluster mass variation with post-explosion time for the $15 M_{\odot}$ progenitor summed over all ejecta zones (from Sarangi \& Cherchneff 2013). 
A. Sarangi acknowledges support from the European Science Foundation Eurogenesis programme CoDustmas network funded by the Swiss National Science Foundation.

\section{References}

Barlow, M. J., Krause, O., Swinyard, B. M., et al. 2010, A\&A, 518, L138

Cherchneff, I. \& Dwek, E. 2009, ApJ, 703, 642

Cherchneff, I. \& Dwek, E. 2010, ApJ, 715, 1

Gomez, H. L., Krause, O., Barlow, M. J., et al. 2012, ApJ, 760, 96

Goumans, F. \& Bromley S. T. 2012, MNRAS, 420, 3344

Kotak, R., Meikle, W. P. S., Farrah, D., et al. 2009, ApJ, 704, 306

Liu, W. \& Dalgarno, A. 1995, ApJ, 454, 472

Lucy, L. B., Danziger, I. J., Gouiffes, C., \& Bouchet, P. 1989, in G. Tenorio-Tagle, M. Moles $\&$ J. Melnick (eds.), IAU Colloq. 120 'Structure and Dynamics of the Interstellar Medium', p. 164

Matsuura, M., Dwek, E., Meixner, M., et al. 2011 Science, 333, 1258

Rauscher, T., Heger, A., Hofmann, R. D., \& Woosley, S. E. 2002, ApJ, 576, 323

Rho, J., Onaka, T., Cami, J., \& Reach, W. 2012, ApJ, 747, 6

Sarangi, A. \& Cherchneff, I., 2013, ApJ, submitted

Spyromilio. J, Meikle, W. P. S., Learner, R. C. M., \& Allen, D. A. 1988, Nature, 334, 327

Sugerman, B. E. K., Ercolano, B., Barlow, M. J., et al. 2006, Science, 313, 196

\section{Discussion}

RAY: Is there an anticorrelation seen between the dust mass and the Ni56 mass?

CherchnefF: The final dust mass found is not truly correlated to the $56 \mathrm{Ni}$ mass, but the time at which dust forms is. The larger the $56 \mathrm{Ni}$ mass, the later dust will form in the ejecta.

Bouchet: 1) What is the limit on the mass of dust? Does it depend of the yields in your model? 2) The original metallicity of the progenitor could be important. If so, which LMC metallicity have you considered for SN87A?

CherchnefF: The dust mass depends on the initial elemental yields but this dependence is not linear, because of the gas phase chemistry and the formation of molecules. We have not yet modeled SN1987A but we would assume a solar metallicity divided by 3 .

UnIDENTIFIED: So what is wrong with the claim of $0.7 M_{\odot}$ of dust in SN 1987A?

CherchnefF: The 0.7 Msun of dust in SN 1987A is an upper limit based on the assumption of $100 \%$ element depletion in dust. The formation of molecules is not taken into account and the bottleneck represented by the nucleation phase of dust is not considered. This number is by no means a dust mass formed in the ejecta.

Koo: I wonder if there is a possibility that crystalline silicates form in cooling SN ejecta

CherchnefF: To go from amorphous to crystalline silicates, you need to maintain a high gas temperature for a long time. These conditions can be found in discs. These conditions are not met in supernova ejecta because the time scale for dust formation is of the order of a few years only. This time is too short for forming crystalline silicates. 